\title{
Influence of nearest-neighbor Coulomb interactions on the phase diagram of the ferromagnetic Kondo model
}

\author{
A. L. Malvezzi, ${ }^{*}$ S. Yunoki, and E. Dagotto \\ National High Magnetic Field Laboratory and Department of Physics, Florida State University, Tallahassee, Florida 32306
}

(Received 21 September 1998)

\begin{abstract}
The influence of a nearest-neighbor Coulomb repulsion of strength $V$ on the properties of the ferromagnetic Kondo model is analyzed using computational techniques. The Hamiltonian studied here is defined on a chain using localized $S=1 / 2$ spins, and one orbital per site. Special emphasis is given to the influence of the Coulomb repulsion on the regions of phase separation recently discovered in this family of models, as well as on the double-exchange-induced ferromagnetic ground state. When phase separation dominates at $V=0$, the Coulomb interaction breaks the large domains of the two competing phases into small "islands" of one phase embedded into the other. This is in agreement with several experimental results, as discussed in the text. Vestiges of the original phase separation regime are found in the spin structure factor as incommensurate peaks, even at large values of $V$. In the ferromagnetic regime close to density $n=0.5$, the Coulomb interaction induces tendencies to charge ordering without altering the fully polarized character of the state. This regime of "charge-ordered ferromagnetism" may be related with experimental observations of a similar phase by Chen and Cheong [Phys. Rev. Lett. 76, 4042 (1996)]. Our results reinforce the recently introduced notion [see, e.g., S. Yunoki et al., Phys. Rev. Lett. 80, 845 (1998)] that in realistic models for manganites analyzed with unbiased many-body techniques, the ground state properties arise from a competition between ferromagnetism and phase-separation-charge-ordering tendencies. [S0163-1829(99)09709-X]
\end{abstract}

\section{INTRODUCTION}

The study of manganite materials continues attracting considerable attention due to their potential technological applications and interesting physical properties dominated by its colossal magnetoresistance effect. ${ }^{1}$ Ferromagnetism in models for manganites has been studied since the 1950s, and it is widely assumed that the double-exchange (DE) ideas are sufficient to understand the stabilization of a magnetic state at low-temperatures upon hole-doping the manganites. ${ }^{2}$ The main reason for this stabilization is that the kinetic energy of the holes is substantially improved in a fully aligned spin background. These concepts are similar to those that lead to the well-known Nagaoka phase in the context of the $t-J$ model for the cuprates. However, experimental work has revealed a phase diagram for manganites that is far more complicated than simple DE models predict. ${ }^{3}$ In particular, at densities corresponding to the undoped compound [referred to as "half-filling" $(n=1)$ in the language of the one-orbital model] an A-type antiferromagnet (AF) is stabilized. After a small amount of holes is introduced, some manganites enter a regime where ferromagnetic droplets have been recently observed. ${ }^{4}$ Upon further doping the DE ferromagnetic metallic regime is reached, including the widely studied $x \sim 1 / 3$ concentration. In the other limit of intermediate- and smallelectron densities, a charge-ordered state is stabilized in Cadoped manganites. ${ }^{3}$ In addition, at densities corresponding to the DE ground state at low temperatures, a metal-insulator transition occurs as the temperature is raised. This insulating state is likely responsible for the colossal magnetoresistance effects in manganites.

Recent work has initiated the computational analysis of models for these compounds, ${ }^{5-8}$ allowing for calculations that go beyond simple mean-field approximations. The techniques used for such computational studies are borrowed in part from the field of cuprates, where they were applied to the analysis of $t-J$-like models in recent years. ${ }^{9}$ The use of unbiased techniques in models for manganites has already led to a fairly good understanding of the zero-temperature phase diagram of the so-called Ferromagnetic Kondo model (FKM) which is based on the assumption that only one $e_{g}$ orbital is relevant. Although Jahn-Teller (JT) effects cannot be neglected in a quantitative study of manganites, ${ }^{10}$ it is important to clarify the properties of this simplest one-orbital model before addressing more complicated two-orbital Hamiltonians with JT phonons.

Following this computational approach, Yunoki and co-workers ${ }^{5-7}$ recently reported the phase diagram of the FKM addressing dimensions $D=1,2,3$, and $\infty$. The computational techniques used were the Monte Carlo method in $D=1,2,3$ with classical $t_{2 g}$ spins, the dynamical mean-field approximation in $D=\infty,{ }^{11}$ as well as the exact diagonalization (ED) and density-matrix renormalization group (DMRG) (Ref. 12) techniques in one dimension for quantum-mechanical localized $t_{2 g}$ spins. The phase diagram was found to contain three dominant regimes: (i) a ferromagnetic phase induced by the DE mechanism, (ii) a spin incommensurate regime at intermediate and small Hund coupling ${ }^{13}$ predominantly due to the Ruderman-Kittel-Kasuya-Yosida (RKKY) interaction, and (iii) a region of "phase separation" (PS) near density $n=1$ between ferromagnetic hole-rich and antiferromagnetic hole-undoped phases. The latter regime (PS) is unexpected since for a long time it has been assumed that the transition from the $\mathrm{AF}$ regime, characteristic of the 
undoped region, to the FM regime at $x \sim 1 / 3$ should occur through a spin-canted state. ${ }^{14}$ However, the results of Ref. 5 showed that this is incorrect and actually the transition from AF to FM occurs through the creation of "islands" of one phase embedded into the other, growing in size as the overall density changes. Work by other groups ${ }^{15}$ is in agreement with the main conclusions of Ref. 5. Previous studies in onedimensional (1D) models also indicated the relevance of phase separation as one moves from copper to the left in the transition-metal row of the periodic table of elements. ${ }^{16}$

On the experimental side, a growing body of evidence suggests that the transition from undoped $\mathrm{LaMnO}_{3}$ to the doped ferromagnetic compounds indeed occurs through an inhomogeneous process. For instance, the existence of quasistatic magnetic droplets at small hole-density in $\mathrm{La}_{1-x} \mathrm{Ca}_{x} \mathrm{MnO}_{3}$ has recently been emphasized using elastic neutron scattering techniques at $x=0.05$ and 0.08 below the critical temperature for the magnetic state. ${ }^{4}$ Phase separation using NMR techniques has also been observed for the same compound at low temperatures. ${ }^{17}$ Working at $x=1 / 3$ and at temperatures above the ferromagnetic critical temperature $T_{c}^{\mathrm{FM}}$, neutron scattering experiments were interpreted as providing evidence for two distinct phases in the system, ${ }^{18}$ while small-angle neutron scattering and magnetic susceptibility data suggested the presence of magnetic clusters $(\sim 12 \AA$ in size) in the same regime. ${ }^{19} \mathrm{X}$-ray and powder neutron scattering methods applied to $\operatorname{Pr}_{0.7} \mathrm{Ca}_{0.3} \mathrm{MnO}_{3}$ also revealed the existence of ferromagnetic clusters. ${ }^{20}$ Neutron studies of $\mathrm{La}_{1-x} \mathrm{Sr}_{x} \mathrm{MnO}_{3}$ at $x=0.10$ and 0.15 were interpreted as corresponding to a polaron-ordered arrangement. ${ }^{21}$ Experimental studies of the $2 \mathrm{D}$ compound $\mathrm{Sr}_{2-x} \mathrm{La}_{x} \mathrm{MnO}_{4}$ at small electronic density have also been considered as evidence of phase separation in manganites. ${ }^{22}$ Many other papers have reported results compatible with charge segregation tendencies at temperatures and densities that surround the lowtemperature ferromagnetic phase of the manganites. ${ }^{23-25}$

This interesting agreement between theory and experiments reinforces the notion that simple electronic models for manganites with a strong Hund coupling may contain the essence of the physics needed to describe these materials. However, to confirm this assumption it is important to proceed further with the computational calculations in two main directions: (i) first, the influence of Jahn-Teller phonons and the use of two $e_{g}$ orbitals are important for a qualitative comparison between theory and experiment. An effort in this direction has been recently reported by two of the authors and Moreo. ${ }^{8}$ The existence of PS with JT phonons was confirmed, including a tendency to a novel "orbital" phase separation regime. ${ }^{8}$ PS tendencies were found to be as robust in the phase diagram as in the case of a one-orbital Kondo model. This shows that studies of one-orbital Hamiltonians can capture at least part of the physics of more sophisticated multi-orbital models for manganites; (ii) second, Coulomb interactions beyond the on-site term are important to avoid the accumulation of charge suggested by the phase separation process described in Ref. 5. It is likely that in the region of "unstable" densities the large clusters of the hole-rich ferromagnetic phase will be divided into small clusters due to this Coulomb repulsion. Actually previous work in the context of the cuprates has shown the tendency to form extended charge-ordered structures ${ }^{26}$ once a long-range Cou- lomb interaction is added to a phase separated state.

It is precisely one of the purposes of the present paper to study the influence of Coulomb interactions beyond the onsite $U$ term on a model for manganites that has the tendency to phase separate in its ground state. The selection of the model for the present investigation is important for technical reasons. The ferromagnetic Kondo model with classical $t_{2 g}$ spins analyzed in Ref. 5 is difficult to study with Coulomb interactions since in this case the Monte Carlo method will need the addition of Hubbard-Stratonovich (HS) degrees of freedom to decouple the four-fermion terms in the Hamiltonian. In addition to the intrinsic complexity of this procedure, the HS decoupling will lead to "sign problems" that likely will prevent the study of low-temperature properties. To complicate matters even more, techniques that deal directly with the Hilbert space of the problem, such as ED and DMRG, cannot be easily applied to a model with classical spins. Then, in order to analyze the influence of intersite Coulomb terms it would be better to use quantum $t_{2 g}$ spins and ED or DMRG methods for their analysis. In this context there are no "sign problems." However, the huge Hilbert spaces that need to be studied in finite size chains impose constraints on the value of the quantum localized spins $S$. In order to carry out a study on a cluster large enough to reach bulk properties $S$ must be restricted to $1 / 2$, rather than the $3 / 2$ realistic value corresponding to manganites. ${ }^{27}$ Previous calculations ${ }^{6}$ have shown that qualitatively $S=1 / 2,3 / 2$, and $\infty$ (classical spins) give very similar phase diagrams in the absence of Coulomb interactions, and there is no reason to expect that this situation will change when these interactions are added. In addition, again due to the large size of the Hilbert spaces involved in the problem our analysis must be restricted to one-dimensional systems. Previous work has shown the existence of many similarities between the results obtained in all dimensions 5 including 1 . Thus, the restriction of working on chains should not be considered severe, and the analysis below is expected to capture the main qualitative aspects of the problem under investigation.

Note that calculations using $S=1 / 2$ localized spins on chains have relevance not only in the context of manganites but also for recently synthesized one-dimensional materials such as $\mathrm{Y}_{2-x} \mathrm{Ca}_{x} \mathrm{BaNiO}_{5}$ which have two active electrons per $\mathrm{Ni}$ ion. This compound has been studied experimentally $^{28}$ and theoretically, ${ }^{29}$ and upon doping interesting properties have been observed including a metalinsulator transition.

To facilitate the understanding of the main results of this paper the phase diagram in one dimension of the Ferromagnetic Kondo model (without Coulombic interactions and using localized $S=1 / 2$ spins) is reproduced in Fig. 1 from Ref. 6. As explained before, three main regimes were found using ED and DMRG techniques. The region labeled FM corresponds to saturated ferromagnetism (the ground state spin is maximized). The IC regime suggests the existence of incommensurate spin correlations, at least of short range, and it manifests itself through the behavior of the spin structure factor $S(k)$ which has a peak that moves away from $k=\pi$ as holes are added to the "half-filled" system. Finally, the regime labeled PS corresponds to phase separation which was studied calculating the density $n$ vs the chemical potential $\mu$, 


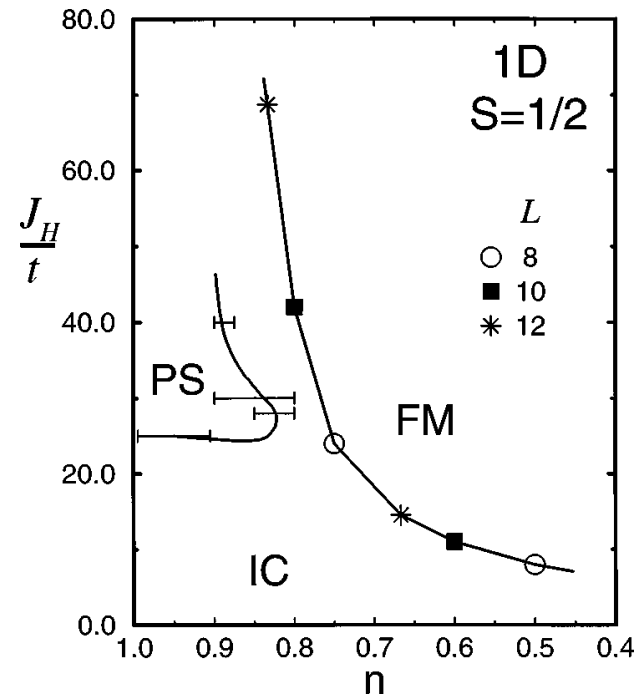

FIG. 1. Phase diagram of the ferromagnetic Kondo model with localized $S=1 / 2 t_{2 g}$ spins. FM, PS, and IC denote regions with saturated ferromagnetism, phase separation, and spinincommensurate correlations, respectively. The results for the FM phase were obtained exactly with the ED technique with up to 12 sites. The rest with the DMRG method with up to 40 sites. The phase diagram is taken from Ref. 6.

as well as the inverse compressibility, searching for unstable densities. ${ }^{6}$

The goal of the present manuscript is to analyze how the phase diagram of Fig. 1 changes when Coulomb interactions are added to the one-orbital Kondo model. The organization of the paper is as follows. In Sec. II the details of the model, technique, and calculated observables are given. In Sec. III the phase diagram is constructed when a nonzero on-site repulsion is considered, but without including nearest-neighbor Coulomb interactions. In Sec. IV these longer-range interactions are added. The tendency to form charge-ordered states (at least at short distances) reported in this section is the main result of this paper. Implications for experiments are discussed in the Conclusions (Sec. V).

\section{MODEL AND COMPUTATIONAL TECHNIQUE}

As explained in the Introduction, the model used in the present study is the one-orbital ferromagnetic Kondo model with localized (spin-1/2) degrees of freedom that mimic the effect of the $t_{2 g}$ electrons. Coulomb interactions in the $e_{g}$ band are also incorporated in the model. The Hamiltonian defined on a chain with $L$ sites is

$$
\begin{aligned}
H= & -t \sum_{\langle i j\rangle \sigma}\left(c_{i \sigma}^{\dagger} c_{j \sigma}+\text { H.c. }\right)-J_{H} \sum_{i} \mathbf{S}_{i f} \cdot \mathbf{s}_{i c}+J^{\prime} \sum_{\langle i j\rangle} \mathbf{S}_{i f} \cdot \mathbf{S}_{j f} \\
& +U \sum_{i} n_{i \uparrow} n_{i \downarrow}+V \sum_{\langle i j\rangle} n_{i} n_{j} .
\end{aligned}
$$

The first term is the electron-transfer between Mn ions. $\langle i j\rangle$ denotes nearest-neighbor sites and $t$ is the hopping amplitude that will be set to 1 in most of the manuscript. In the second term, $J_{H}>0$ is the ferromagnetic Hund coupling. The spin$1 / 2$ operator for the conduction electron is defined as $\mathbf{s}_{i c}$ $=\Sigma_{\alpha \beta} c_{i \alpha}^{\dagger} \boldsymbol{\sigma}_{\alpha \beta} c_{i \beta}$, while $\mathbf{S}_{i f}$ represents a localized spin-1/2 at site $i$. A strong Hund coupling will be used throughout the paper, and its value will be fixed to $J_{H}=40$, in units of $t$, unless otherwise stated. $J^{\prime}$ is the strength of a direct Heisenberg coupling between the localized spins. This term is needed on phenomenological grounds since in fully doped manganites (e.g., when all $\mathrm{La}$ has been replaced by $\mathrm{Ca}$ ) a finite Néel temperature is experimentally observed. $U$ is the strength of the on-site electronic repulsion, with $n_{i \sigma}$ the number operator at site $i$ with spin $\sigma$. $V$ regulates the Coulomb repulsion at a distance of one lattice spacing. The rest of the notation in Eq. (1) is standard.

The technique used in this paper to analyze ground state properties of the Hamiltonian Eq. (1) in one dimension is the DMRG method. The finite-system variation of DMRG was used, working with open boundary conditions. ${ }^{30}$ All results were obtained keeping a number of states $m=100$ in the iterations, with the exception of densities $n=1$ and 0 where a smaller number of states produced accurate enough results. With this value of $m$ a truncation error of order $10^{-6}$ or smaller was obtained throughout the results shown in the next sections.

In order to characterize the ground state properties of Eq. (1), a variety of expectation values have been calculated. The spin structure factor defined as

$$
S(k)=\frac{1}{L} \sum_{j, m}\left\langle\mathbf{S}_{j f} \cdot \mathbf{S}_{m f}\right\rangle e^{i(j-m) k}
$$

and the charge structure factor

$$
N(k)=\frac{1}{L} \sum_{j, m}\left\langle n_{j} n_{m}\right\rangle e^{i(j-m) k}
$$

are among the measured quantities. In addition, the inverse compressibility defined as

$$
1 / \kappa=\frac{N^{2}}{4 L}[E(N+2, L)+E(N-2, L)-2 E(N, L)],
$$

was also calculated. Here $E(N, L)$ is the ground state energy of a chain of $L$ sites with $N$ electrons, and density $n=N / L$. Finally, the charge correlation $C(i)=\left\langle n_{j} n_{m}\right\rangle-\left\langle n_{j}\right\rangle\left\langle n_{m}\right\rangle$, where $i=|j-m|$, was studied in some special cases. Here the angular brackets not only denote expectation value in the ground state but also indicate that for a given distance $i$ all possible pairs of sites $j, m$ of the cluster compatible with $i$ $=|j-m|$ have been used. The reason is that open boundary conditions are needed in the DMRG technique and, thus, the correlations are different at, e.g., the center and near the chain end. Such an average procedure uses information from the whole chain, and in practice it produces smoothly changing results as the distance $i$ and the couplings are varied.

\section{RESULTS AT $V=0$}

Let us begin the study for the case without a repulsion at a distance of one lattice spacing (i.e., working at $V=0$ ). The results obtained here will be later compared with those of the following section for $V \neq 0$. 

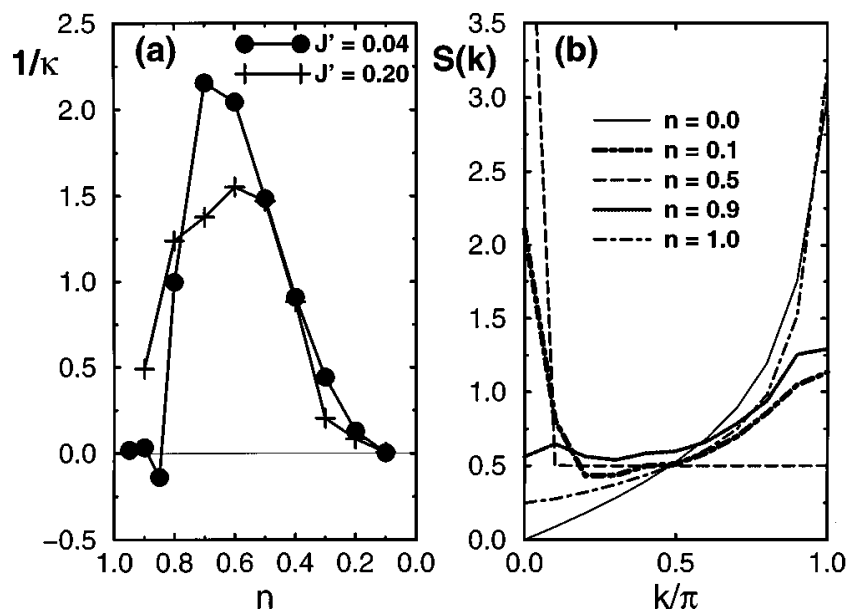

FIG. 2. (a) Inverse compressibility $1 / \kappa$ versus density $n$ for the FM Kondo model with $U=V=0$ and two values of $J^{\prime}$, as indicated. The results were obtained using chains with 20 and 40 sites. For $J^{\prime}=0.041 / \kappa$ is very small or even negative at both extremes of $n$ close to 1 and 0 . At $J^{\prime}=0.20$ the system is unstable only at small density. (b) Spin structure factor $S(k)$ for a variety of densities (indicated), $U=V=0, J^{\prime}=0.04$, and a 20 site chain. For $n$ $=0.1$ and 0.9 , a coexistence of ferromagnetic and antiferromagnetic features is observed. Note that the value 0.5 for $S(k \neq 0)$ in the fully polarized state at $n=0.5$ originates in the particular structure of the spin correlation which gives $3 / 4$ on-site and $1 / 4$ for other distances.

\section{A. Zero on-site repulsion $U$}

In Fig. 2(a), the inverse compressibility $1 / \kappa$ is shown as a function of $n$, with both the on-site and intersite repulsions $U$ and $V$ equal to zero, in order to study the dependence of the results with $J^{\prime}$. A similar analysis was carried out by Yunoki and Moreo $^{7}$ but in the classical localized spin limit. This study found that $J^{\prime}$ is an important parameter in determining the low-temperature properties of models for manganites. Fig. 2(a) shows that $1 / \kappa$ at $J^{\prime}=0.04$ is small or even slightly negative both in the limits of small and large density, in good qualitative agreement with Ref. 7. For larger values of $J^{\prime}$, Fig. 2(a) suggests that the phase separation regime near $n$ $=1$ is lost (i.e., all densities are stable), while the results close to $n \sim 0$ are only slightly affected. Then, in order to study the effect of a nearest-neighbor $(\mathrm{NN})$ repulsion $V$ over a phase separated regime, relatively small values of $J^{\prime} / t$ must be selected. Note that this is not a problem since experimental results have actually shown that $J^{\prime} \sim 0.05$ in units of $t=0.2 \mathrm{eV}$ is a realistic value for the exchange coupling between the localized spins. ${ }^{7,24}$ Then, in the rest of the paper $J^{\prime}=0.05$ will be used, unless otherwise stated.

Let us now analyze the behavior of the spin structurefactor $S(k)$ as a function of density. The results are shown in Fig. 2(b). As expected, in the limits $n=1$ and 0 a clear signal for strong antiferromagnetic correlations is observed since $S(k)$ is peaked at $k=\pi{ }^{31}$ In the intermediate regime where double-exchange tendencies leads to a ferromagnetic ground state, $S(k)$ is maximized at $k=0$ also as expected based on previous literature. The most interesting results in this context arise close to $n=1$ and also 0 . In this regime, $S(k)$ has important weight both at $k=0$ and $\pi$ signaling the coexistence of FM and AF domains as expected in a phaseseparated regime. This is in agreement with the previous

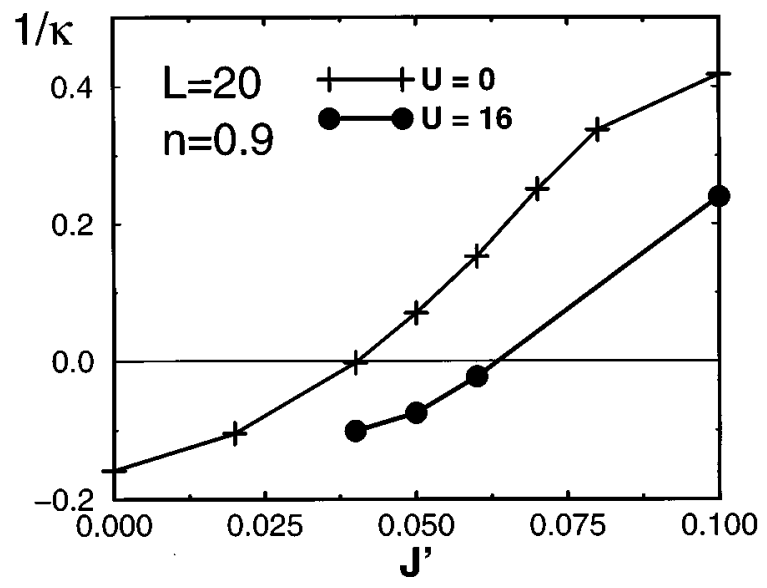

FIG. 3. Inverse compressibility $1 / \kappa$ versus $J^{\prime}$ at $n=0.9, V$ $=0$, using a chain with 20 sites, and two values of $U$ as indicated. The results illustrate that at large Hund coupling, $U$ does not influence much on the ground state properties.

work reported in Ref. 5, with the discussion in the Introduction, and with the inverse compressibility data shown in Fig. 2(a). Note that the densities $n$ that correspond to the phase separation regime can be studied in detail since the analysis presented here is in the canonical-ensemble formalism. If a grand-canonical approach would be used, as in Ref. 5, then the regimes $n \sim 0.1$ and $\sim 0.9$ would not be reachable.

\section{B. Nonzero on-site repulsion $U$}

For the Hund coupling used throughout this paper, moderate values of the on-site Coulomb interaction $U$ are not expected to play an important role since double occupancy is naturally suppressed by a large $J_{H}$. To illustrate this statement, in Fig. 3 the inverse compressibility is shown at $n$ $=0.9$, namely, in a regime with phase separation at $U=V$ $=0$, as discussed in the previous subsection. When $U$ is switched on and increased to a large value in units of the hopping amplitude $(U=16)$, the plots of $1 / \kappa$ vs $J^{\prime}$ change only slightly. As a consequence, in the rest of the paper $U$ will be fixed to 16 to avoid the proliferation of free parameters. The conclusions of our paper are not expected to change as long as $J_{H}$ and $U$ are the largest scales in the problem.

The compressibility at $V=0$ and $U=16$ as a function of $n$ is shown in Fig. 4(a). It is clear that both at small and large $n$, this quantity is either $\sim 0$ or negative signaling the instability of the ground state towards the formation of two different phases. In between, where the system is expected to be ferromagnetic, the ground state is stable. In Fig. 4(b) the spin structure factor $S(k)$ is shown to illustrate the coexistence of FM and AF features in the ground state of the "unstable", regime. This occurs at $n=0.85$ where peaks at both $k=0$ and $\pi$ are observed. In the other cases, $n=1$ and 0.75 , there is only one dominant peak at the antiferromagnetic and ferromagnetic locations, respectively.

To further confirm if at small and large $n$ phase separation is indeed observed, the on-site densities $\left\langle n_{i}\right\rangle$ (expectation value in the ground state of the local density operator) have also been monitored. Note here that the DMRG method works using open boundary conditions and, as a conse- 

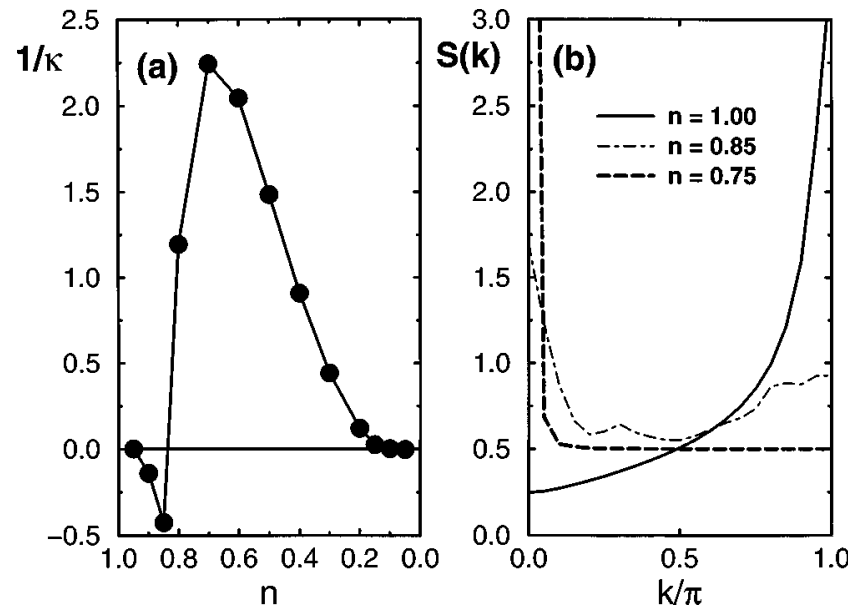

FIG. 4. (a) Inverse compressibility $1 / \kappa$ vs density $n$, working at $J_{H}=40, U=16, V=0, J^{\prime}=0.05$, and using 20 and 40 sites. The results show that near $n \sim 1$ and $n \sim 0$ phase separation occurs, as shown in Fig. 2(a) even including a large on-site repulsion $U$. (b) Static spin structure factor $S(k)$ vs momentum $k / \pi$ for the same $J_{H}, U, V$ couplings used in (a), $J^{\prime}=0.05,40$ sites, and several densities. The result at $n=0.85$ illustrates the coexistence of FM and AF features.

quence, the on-site density changes from site to site. In Fig. 5 (a) results at $n=0.75$, where the compressibility is positive and $S(k)$ peaks at $k=0$, show that aside from a boundary effect involving about three sites at each chain end the local density only slightly Friedel oscillates around the global density. On the other hand, in the presumed to be phaseseparated regime the local density changes substantially as a function of the site position $i$. Most of the holes are near the boundary and the local density changes abruptly between two values, which are the extreme stable densities $\sim 0.75$ and $\sim 1$ as expected. Also the charge structure factor $N(k)$ [Fig. 5 (b)] has the characteristics corresponding to phase separation, namely at $n=0.75$ it behaves as a noninteracting spin-
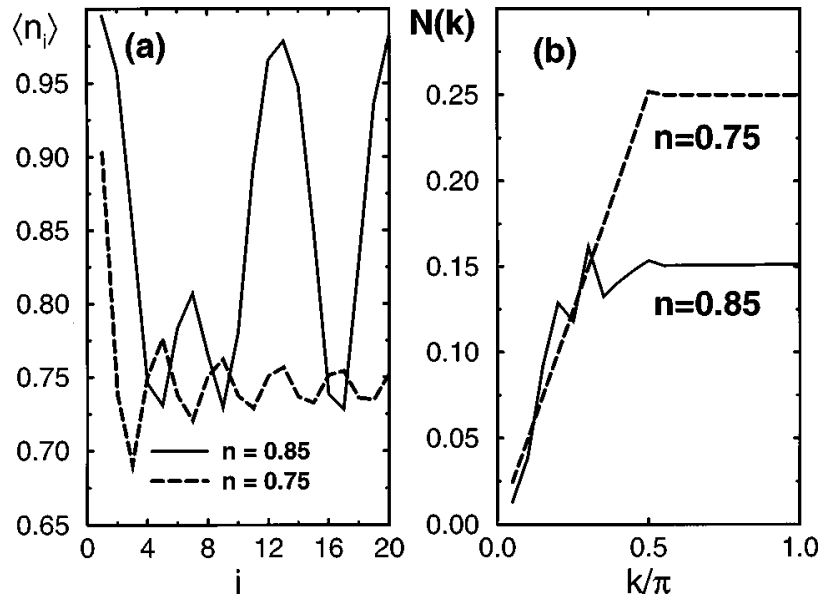

FIG. 5. (a) Local density $\left\langle n_{i}\right\rangle$ vs position along the chain $i$ for the same couplings as in Fig. 4(a). The chain length is 40.1 is the first site of the chain starting from the left. 20 is the center of the chain. The results for $i>20$ are obtained by reflection. Shown are data for $n=0.75$, representative of the ferromagnetic region, and $n=0.85$, which belongs to the phase separated regime. (b) Charge structure factor $N(k)$ at the two densities used in (a).
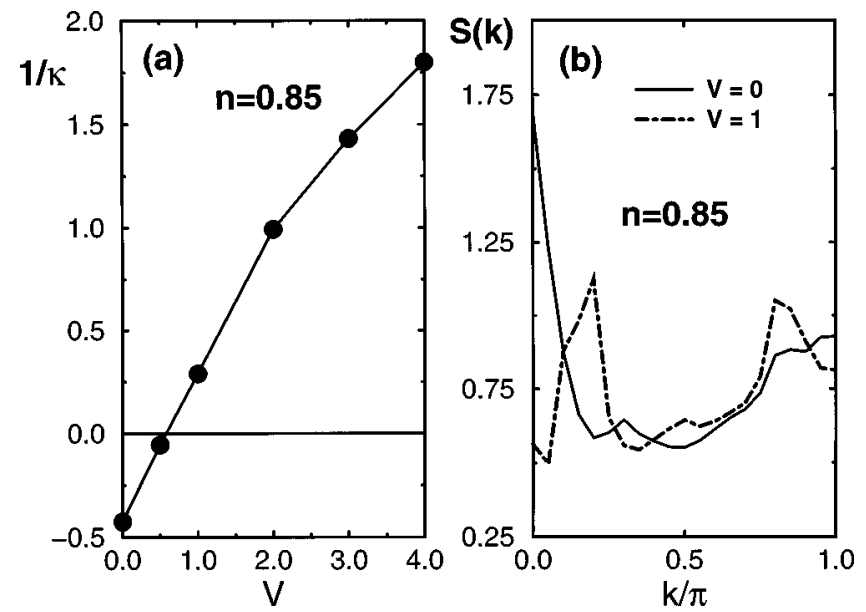

FIG. 6. (a) Inverse compressibility $1 / \kappa$ vs $V$ working at $J_{H}$ $=40, U=16, J^{\prime}=0.05$ and density $n=0.85$ (i.e., in the region of phase separation for $V=0$ ). Shown are results using 40 sites. $1 / \kappa$ becomes positive for an intersite Coulomb interaction $V \sim 0.5$ making the ground state stable. (b) $S(k)$ for the parameters used in (a) and two values of $V$ (actually results for $V>1$ are similar to those of $V=1)$.

less fermionic system due to the ferromagnetic character of the ground state, while at $n=0.85$ it develops structure at small wave numbers related with the inhomogeneous distributions of charge.

In short, the results of this subsection have shown that the Kondo model with localized $S=1 / 2$ spins, and with the addition of a $J^{\prime}$ coupling among them, has a qualitative phase diagram similar to the results presented before in the classical limit for the $t_{2 g}$ spins in Refs. 5 and 7. This conclusion does not change even if an on-site repulsion $U$ of moderate strength is incorporated in the problem. Then the present analysis has allowed us to fix the parameters $J_{H}, U, J^{\prime}$ such that the physics under investigation, focused on phase separation and ferromagnetism, is realized in the ground state of the model Eq. (1). Thus, the problem is now ready for the analysis of the influence of $V$ on the phase diagram.

\section{RESULTS INCLUDING A REPULSION $V$}

\section{A. Influence of $V$ on the phase separated regime}

The $V$ term will now be switched on at a density such that phase separation occurs in the ground state. In Fig. 6(a) the inverse compressibility is shown as a function of $V$ using a 40 -site chain. The results show that the unstable region observed at $V=0$ in the previous section now becomes stable when $V>0.5$. Then, in agreement with the introductory discussion, phase separation is severely affected by Coulombic interactions beyond the on-site term. However, here it is interesting to observe that vestiges of phase separation survive even up to large couplings $V$. For instance, consider $S(k)$ which is shown in Fig. 6(b). At $V=1$, a double-peaked structure is observed, as it occurs at $V=0$, but now with maxima deviated from 0 and $\pi$ forming incommensurate structure. Results at other values of $V>1$ (not shown) are very similar to those at $V=1$. Then, the ground state properties do not seem to change abruptly with $V$ but smoothly. Even ground states that have been stabilized by the Coulomb interaction in 

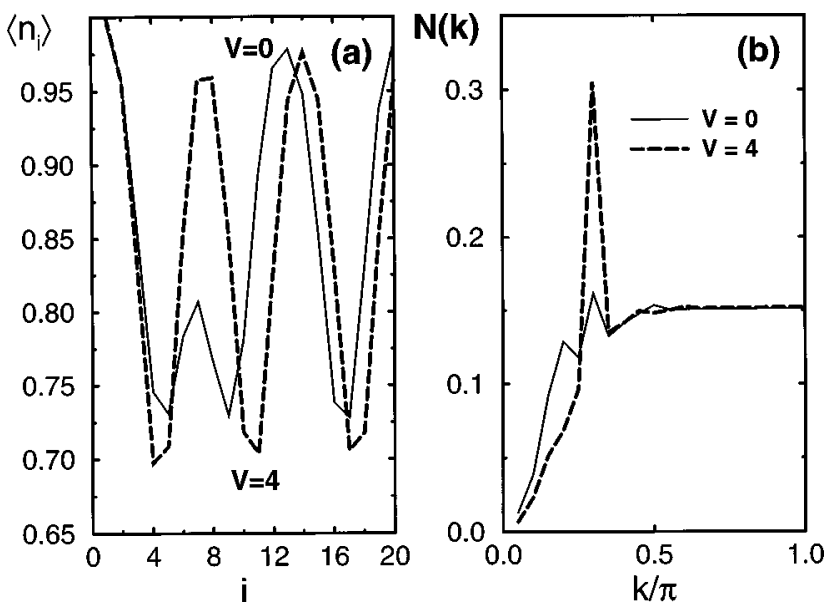

FIG. 7. (a) Local density $\left\langle n_{i}\right\rangle$ vs position along the chain $i$ at $n=0.85$. For the site convention see the caption of Fig. 5(a). The couplings and density are the same as in Fig. 6(a). The solid (dashed) line represents results for $V=0 \quad(V=4)$. (b) $N(k)$ for the two values of $V$ used in (a). The rest of the couplings and density are as in (a).

this regime contain a spin structure-factor with remnants of FM and AF domains. It is only the macroscopic accumulation of charge that is penalized by $V$.

In Fig. 7(a) the local density is shown for the case of six holes on a 40-site chain. The accumulation of charge near the boundary characteristic of phase separation at $V=0$ is replaced by a fairly clear periodic distribution of charge at $V$ $=4$. This occurs not only at $V=4$ but in a wide range of couplings. The replacement of phase separation by a state with charge-ordering tendencies was expected based on the discussion given in the Introduction. The density where the "holes" are mostly located is close to 0.7 . There is no clustering of charge at large $V$. From Fig. 7(b) it can be observed that the charge structure-factor $N(k)$ at $V=4$ develops a very sharp peak at $k=2 \pi n$ due to the periodic arrangement of charge in the ground state.

It is interesting to notice that the positions where the "holes" are in Fig. 7(a) $(V=4)$ are actually made out of four sites instead of one. Then, the holes are not fully static but they have some mobility, and it is natural that to enhance this mobility the spins must be aligned. Then, the large FM regions at small $V$ in the regime of phase separation are now replaced by periodically distributed small regions resembling magnetopolarons. This is among the most important results discussed in this paper.

\section{B. Influence of $V$ on the ferromagnetic regime}

After analyzing in the previous subsection the influence of $V$ on the properties of a phase-separated ground state near $n=1$, let us study what occurs when a NN-Coulomb interaction is switched on in a fully ferromagnetic ground state. Such a state can be easily obtained in the present model Eq. (1) simply using a density, e.g., $n=0.6$ where the doubleexchange ideas are operative. The results for $S(k)$ are shown in Fig. 8(a). While at $V=1$ (and smaller values) the peak at $k=0$ characteristic of ferromagnetism remains strong, at $V$ $=8$ it has reduced substantially its intensity and moved
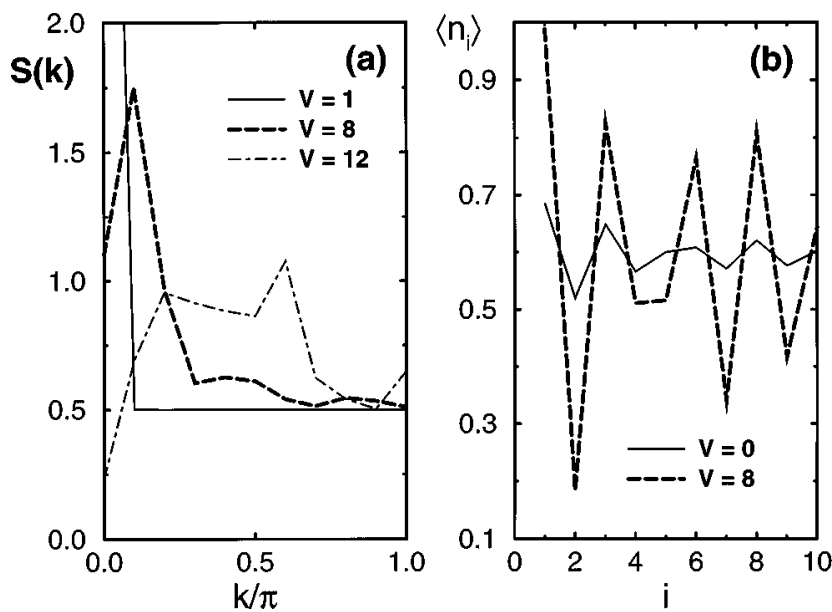

FIG. 8. (a) Spin structure factor $S(k)$ working with $J_{H}$ $=40, U=16, J^{\prime}=0.05, n=0.6,20$ sites, and the values of $V$ indicated. (b) Local density $\left\langle n_{i}\right\rangle$ for the same couplings and density as in (a). Results for a chain of 20 sites are shown. $i=1$ is the site at the extreme left of the chain. The results for $i>10$ are obtained by reflection of the numbers shown. The results at $V=0$ are almost uniform while at $V=8$ they present clear large oscillations.

slightly from $k=0$. At $V=12$ a very broad peak is the only remnant of the ferromagnetic structure at small $V$.

To understand the reason for the complicated behavior of $S(k)$, the local density at $n=0.6$ is shown in Fig. 8(b). At $V=0$ it is almost uniform as expected in a ferromagnetic state. However, at $V=8$ a charge-ordered pattern is observed. This is in agreement with the previously discussed results at $n=0.85$ which showed a similar tendency. However, note that now most minima in the local density involves only one site, instead of four as in Fig. 7(a). For this lattice and number of electrons (12 of them on a 20 -site chain), the charges cannot arrange themselves in a periodic structure, causing the incommensuratelike peak in $S(k)$ at $V=8$. The replacement of a metallic FM state by a state with charge-ordering tendencies is also clear in Fig. 9(a) where $N(k)$ is shown. At $V=8$ a large peak is observed at $k$ $=2 \pi n$. Also the real-space density-density correlations $C(i)$ shown in Fig. 9(b) (for its definition see Sec. II) indicate strong effects in the charge sector at short distances: while at $V=0$ these correlations are not negligible only at distances $i=0$ and 1 , at $V=8$ the same correlations have been enhanced and they are now robust also at distances 2 and 3.

Working at exactly $n=0.5$ the incommensurate structures observed at $n=0.6$ should disappear. In Fig. 10(a) the local density is shown for a 20-site cluster and several values of $V$. At $V=0$ the density is exactly uniform for symmetry reasons, but as soon as $V$ is switched on a charge-ordered pattern clearly emerges. At this density a soliton at the center of the chain appears justifying the reduction in $\left\langle n_{i}\right\rangle$ towards that center. $N(k)$ in Fig. 10(b) also presents a clear peak at $k=\pi$ which grows with $V$, in agreement with the previous discussion. Then, from the analysis of the densities $n=0.6$ and 0.5 it is concluded that a tendency to charge ordering, at least at short-distances, is obtained once an intersite Coulomb interaction $V$ is introduced in a fully ferromagnetic state. This state [which here is referred to as charge-ordered (CO) for simplicity although the large distance behavior is 

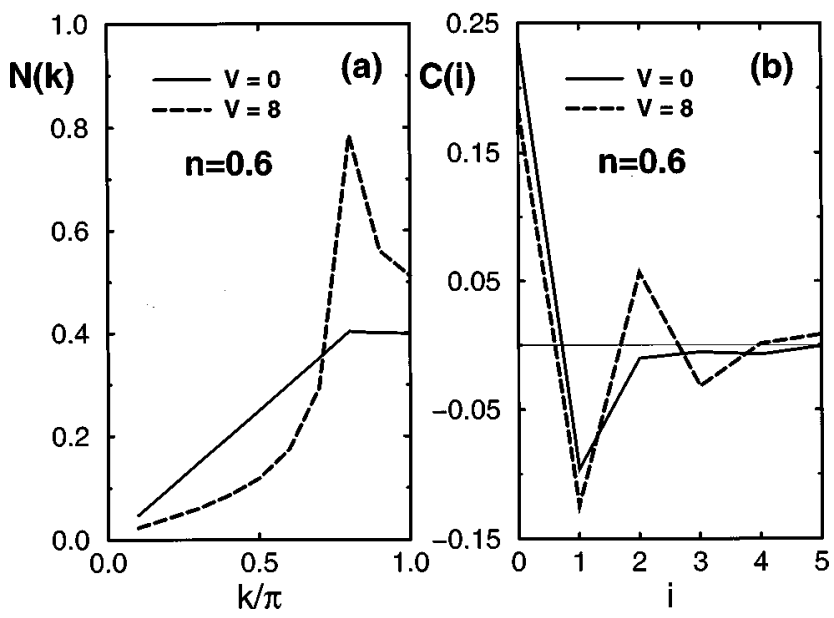

FIG. 9. (a) $N(k)$ vs $k / \pi$ for the couplings $J_{H}, U, J^{\prime}$ and density of Fig. 8(a), and two values of $V$ (indicated). The chain has 20 sites. At $V=8$ the large peak suggests strong charge correlations in the ground state. (b) Density-density correlations $C(i)$ (for its definition see text) vs distance $i$, at the same couplings, chain length, and density used in (a). Enhancement of charge correlations at $V=8$ is observed.

difficult to analyze] occurs within the ferromagnetic phase, i.e., through a calculation of $S(k)$ the spins were found to be fully polarized unless $V$ reaches larger values than those shown in Figs. 10(a) and 10(b). A similar result was reported in Ref. 32 using a path-integral approach.

In order to monitor the development of charge correlations in the ground state the mean value of $n_{c d}=\Sigma_{i=\text { odd }}\left(n_{i}\right.$ $\left.-n_{i+1}\right)$ has been studied before in other models. ${ }^{33}$ Its expectation value should be zero in a metallic state, but nonzero in a system with charge order. Results for a chain of 20 sites are shown in Fig. 11(a). Although nonzero for all values of $V$ $\neq 0$, this result suggests only a tendency towards the formation of a charge-ordered state. For a proper analysis of the
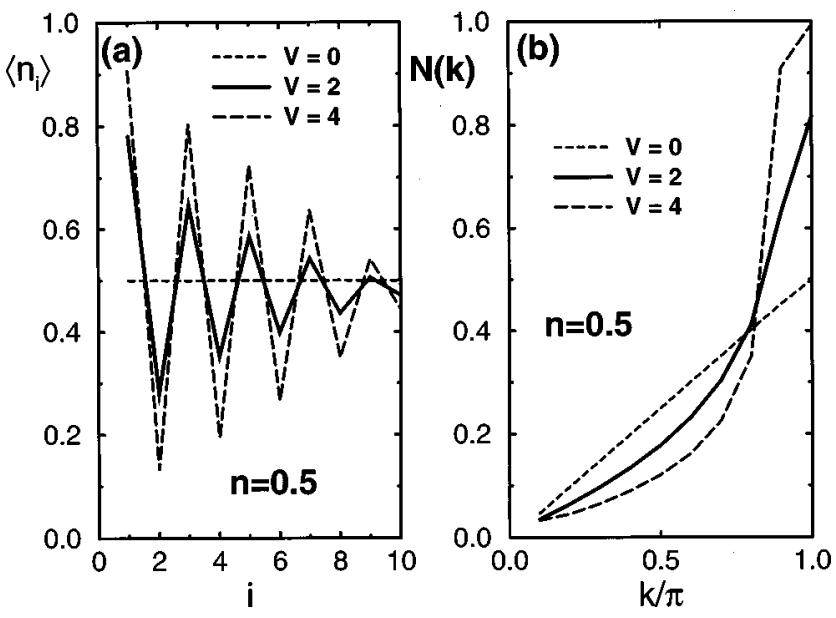

FIG. 10. (a) Local density $\left\langle n_{i}\right\rangle$ corresponding to a 20-site chain with $J_{H}=40, U=16, J^{\prime}=0.05, n=0.5$, and the values of $V$ indicated in the figure. The development of charge oscillations as $V$ grows is clear in the figure. (b) $N(k)$ vs $k / \pi$ using the same couplings, chain length, and density as in (a). The development of a large peak at $k=\pi$ is also compatible with the stabilization of a charge ordered pattern.
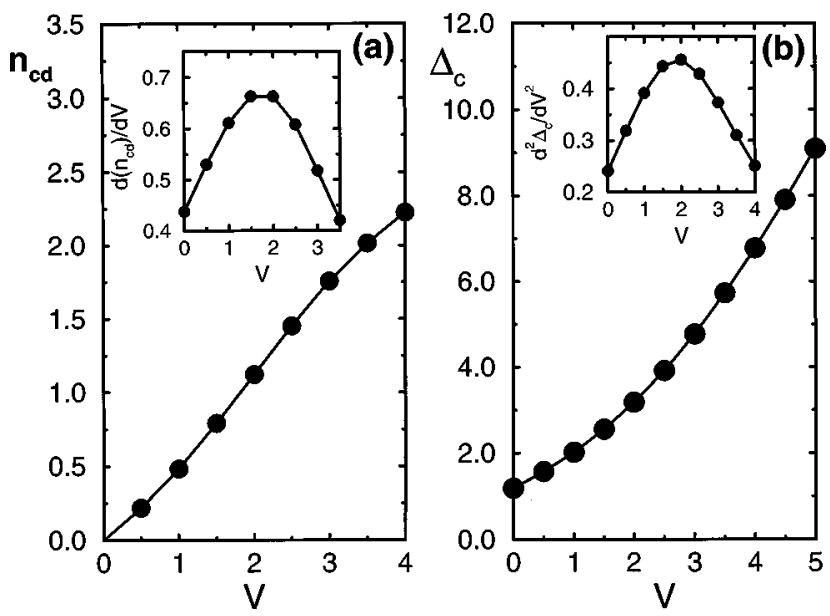

FIG. 11. (a) $n_{c d}$ (see text) vs $V$ for the same $J_{H}, U, J^{\prime}$, chain length, and density as in Fig. 10(a). In the inset the first derivative is shown. (b) Charge gap $\Delta_{c}$ as defined in text vs $V$. The couplings, chain length, and density are as in (a). The inset shows the second derivative. For a discussion see text.

critical $V$ leading to charge ordering a careful finite-size study is needed, beyond the scope (and numerical accuracy) of the present paper. However, in order to gain at least some insight from the 20-site cluster, the first derivative of $n_{c d}$ with respect to $V$ is shown in the inset of Fig. 11(a). Previous calculations studying variations around the $t-J$ model showed $^{33}$ that the critical point is located at the inflection point of the $n_{c d}$ vs $V$ curve. The calculation shown in Fig. 11(a) suggests that the critical coupling is roughly estimated to be at $V_{c} \sim 2$, which is in good agreement with the critical coupling corresponding to a model of spinless fermions with $\mathrm{NN}$ repulsion. ${ }^{34}$ The charge gap $\Delta_{c}(N, L)=E(N+2, L)$ $+E(N-2, L)-2 E(N, L)=4 L / N^{2} \kappa$ was also studied [Fig. 11(b)]. The second derivative of this quantity with respect to $V$ is also peaked around $\sim 2$, as in the case of $d n_{c d} / d V$. Then, this (rough) analysis suggests that a critical coupling $V_{c} \sim 2$ separates two ferromagnetic regions, one charge disordered and the other with at least short-distance chargeordering tendencies. However, further work is needed to establish $V_{c}$ more accurately.

\section{PHASE SEPARATION IN THE SMALL ELECTRONIC DENSITY REGION}

In the low $e_{g}$-density region, a nearest-neighbor repulsion is not expected to affect the ground state properties substantially since the mean distance between electrons is large. Coulombic interactions at distances larger than one lattice spacing would be more important in this regime (but they are difficult to study with the DMRG method). To verify that indeed $V$ is not playing an important role at small $n$, in Fig. 12(a) the inverse compressibility is shown for three values of $V$. While phase separation at large density $n \sim 1$ is affected by $V$ (as discussed in previous sections) and $\kappa$ changes substantially at intermediate densities, the results at low density $n \leqslant 0.20$ are almost $V$ independent.

Let us investigate the properties of the phase-separated regime at small $n$ and $V=0$. The results for the spin structure factor are shown in Fig. 12(b). At $n=0.20$, a clear signal of 

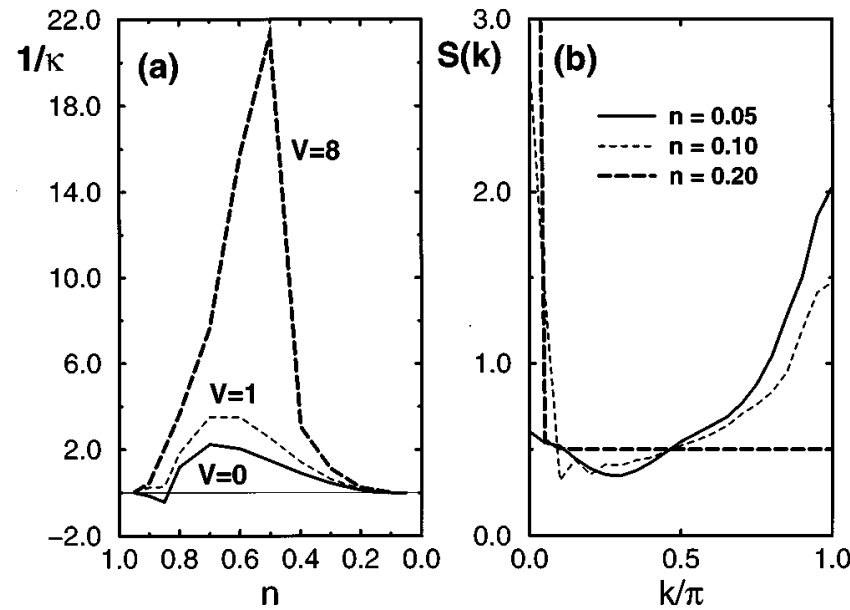

FIG. 12 . (a) Inverse compressibility $1 / \kappa$ vs density $n$, using $J_{H}$ $=40, U=16, J^{\prime}=0.05$, and the three values of $V$ indicated. The numbers are obtained using chains with 20 and 40 sites. The results show that the compressibility is very large at low densities. $V$ has virtually no influence on this region of the phase diagram. (b) $S(k)$ for the same couplings $J_{H}, U$, and $J^{\prime}$ used in (a), a chain of 40 sites, and using $V=0$. The densities are shown. The results at $n$ $=0.10$ and 0.05 illustrate the coexistence of FM and AF features.

ferromagnetism is observed, with $1 / \kappa$ being slightly positive (stable). However, at $n=0.10$ and 0.05 a coexistence of weight both at $k=0$ and $\pi$ appears, signaling the expected regime that separates (i) electron-rich spin-ferromagnetic and (ii) electron-undoped spin-antiferromagnetic states. The presence of PS can also be inferred from the local density $\left\langle n_{i}\right\rangle$ shown in Fig. 13(a). At the stable density $n=0.25$, and leaving aside boundary effects involving about three sites at each end, the density presents Friedel oscillations around the density $n$ [actually the results at this density could have been obtained directly from those of Fig. 5(a) at $n=0.75$ since in
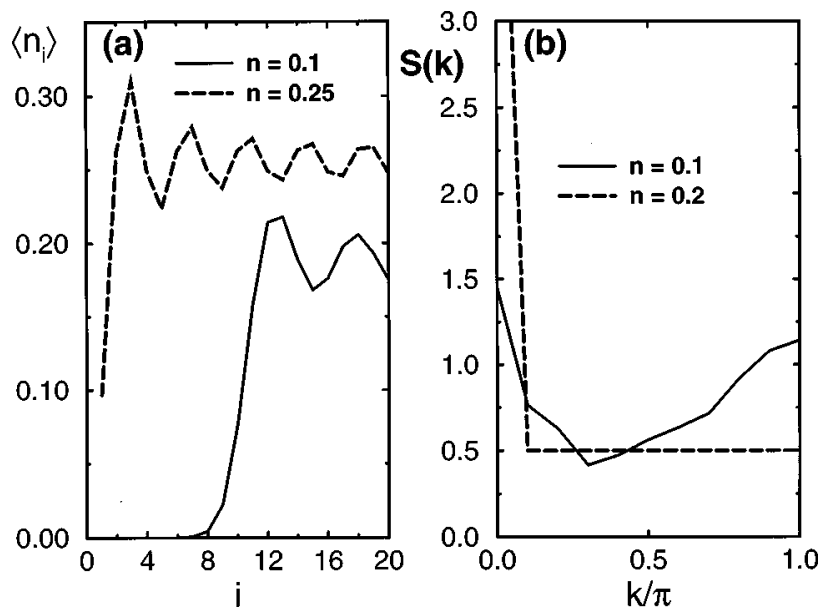

FIG. 13. (a) Local density $\left\langle n_{i}\right\rangle$ for the same couplings $J_{H}, U, J^{\prime}$ used in Fig. 12(a), and $V=0$. The chain used has 40 sites. Results are shown for $n=0.25$ where the densities are very similar (leaving aside a boundary effect near $i=1$ ), and at $n=0.10$ where charge accumulates at the center due to phase separation tendencies in the ground state. (b) $S(k)$ at $J_{H}=40, U=16, J^{\prime}=0.05, V=8$, and for the two densities indicated. The number of sites is 20 . The result at $n=0.1$ shows that even including a large $V$, the coexistence of FM and $\mathrm{AF}$ features persists.

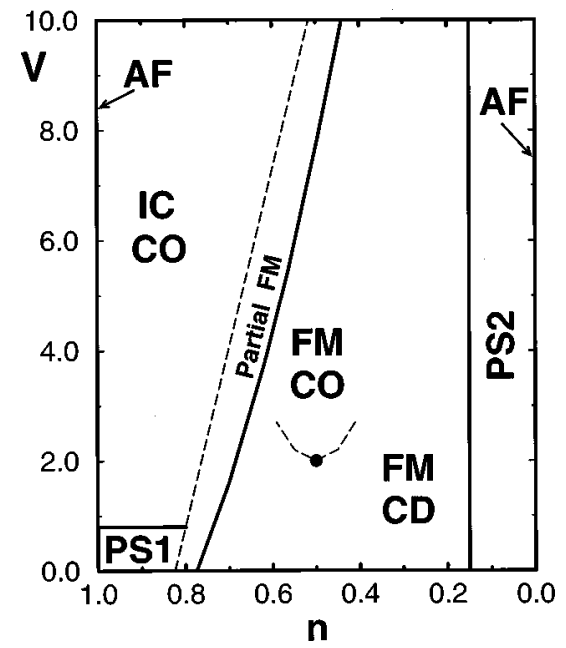

FIG. 14. Phase diagram of the Ferromagnetic Kondo model with on-site and intersite Coulombic interactions, using $S=1 / 2$ localized spins, $J_{H}=40, J^{\prime}=0.05, t=1$, and in one dimension. The results are semi-quantitative since only a finite number of densities can be achieved with clusters of 20 and 40 sites. Thus, small scale oscillations have been smeared out in constructing this figure. PS1 denotes the regime of phase separation at $n \sim 1$, while PS2 indicates a similar regime but now at $n \sim 0$. Both extremes $n=1$ and $n=0$ have strong antiferromagnetic (AF) correlations as indicated. FM denotes a regime where the spin is fully saturated (the total spin is maximum). Partial FM is a regime where the total spin is between the maximum compatible with the cluster and density used, and the minimum (zero). IC indicates a tendency to form spinincommensurate structures (for a discussion see text). CD means "charge disordered." Reciprocally, CO means "charge ordered," which in this case only means that in the ground state strong shortdistance tendencies to charge ordering have been identified (the large distance behavior remains to be analyzed). The point and dashed line at $n=0.5$ and $V=2$ should only be considered as a tentative rough boundary between the $\mathrm{CD}$ and $\mathrm{CO}$ regimens. It represents the evidence discussed in Fig. 11 of a singularity in some observables separating the $\mathrm{CD}$ and $\mathrm{CO}$ regions within the fully saturated ferromagnetic state.

the fully spin-aligned state low and high densities are exactly related by symmetry]. However, at $n=0.10$ the four electrons present in the $L=40$ system accumulate at the center, leaving about ten sites (a quarter of the lattice) virtually empty on each end of the chain. This is the way in which phase separation seems to manifest itself when clusters with open boundary conditions are used. The central cluster of electrons [which has $n \sim 0.2$, i.e., the lower limit of the densities which are stable according to Fig. 12(a)] was found to be spin ferromagnetic as expected. Finally, to further confirm that $V$ does not influence severely on the low-density regime, in Fig. 13(b) $S(k)$ is shown now at $V=8$ for two densities. If $n=0.20$, the system is ferromagnetic, while for $n=0.10$, once again a coexistence of FM and AF features is observed, as in the absence of the intersite Coulomb interaction.

\section{CONCLUSIONS}

In this paper the influence of a nearest-neighbor Coulomb repulsion $V$ between $e_{g}$ electrons was studied using the ferromagnetic Kondo model. The main goal of the paper was to 
analyze the evolution with $V$ of the ground states in the regimes of (i) the recently computationally discovered phase-separation ${ }^{5-7}$ and (ii) with ferromagnetism induced by the double-exchange mechanism. Spin and charge correlations were studied in detail. The computational work using DMRG was made possible by restricting the spatial dimension to 1 , the localized spin value to $1 / 2$, and using only one-orbital per site. These limitations prevent us from making detailed quantitative statements about the effect of Coulomb interactions on real manganite compounds. Actually it is unrealistic to expect that accurate numerical work will be possible in the near future for large enough clusters in dimensions larger than 1 , considering the Coulombic interactions included here. However, several qualitative features have emerged that seem robust enough to survive an increase in dimensionality.

The overall phase diagram in the $V-n$ plane found in this study is presented in Fig. 14. In the limit $V=0$, two regimes of phase separation were observed near $n=1$ (PS1) and 0 (PS2) (in excellent agreement with Ref. 7). In between, a robust ferromagnetic phase was observed with no indications of strong charge ordering tendencies. However, when the Coulomb repulsion $V$ is included in the calculation the PS1 regime rapidly becomes unstable due to the expected energy penalization caused by the charge difference between the two competing phases. At these densities, the $V$ term induces a regular arrangement of charge which resembles a polaron lattice. Each hole is spread over four lattice sites in the regime of parameters investigated in Fig. 7(a). This picture gives support to the intuitive notion that visualizes a phase separated state with extended Coulomb interactions included as a collection of small "islands" of one phase embedded into the other. This result is in good agreement with a large body of experimental work in manganites. ${ }^{4,17-24}$ Vestiges of the phase separated regime are observed in the spin structure factor which has weak incommensuratelike peaks both near $k=\pi$ and 0 . In the other extreme of low electronic density,
PS2 is not affected by $V$ since the mean distance between carriers is large at low density. Here phase separation persists up to large values of $V$. Presumably longer-range Coulombic terms are needed to melt this regime, and induce the chargeordered pattern found in experiments for manganites.

Another of the main results of the paper is the stabilization by NN-Coulomb interactions of a ferromagnetic state with charge periodically distributed at least at short distances. This state is expected to be an insulator but a calculation of the Drude weight is needed to confirm this conjecture. This occurs in the vicinity of $n=0.5$ (Fig. 14). The electrons gain kinetic energy by keeping the spin background fully polarized and they avoid the Coulomb repulsion by forming a charge pattern which is approximately periodic. For $n \neq 0.5$, the charge structure factor peaks away from $k$ $=\pi$, signaling a (natural) tendency to form incommensurate charge arrangements, but its strength is weaker than at $n$ $=0.5$. Then, the present calculation suggests that the ferromagnetic phase of the manganites may coexist with charge ordering tendencies which are maximized at $n=0.5$. Likely these tendencies are more dynamic than static. Note that recent experimental results by Chen and Cheong (Ref. 35) have reported the existence of weakly incommensurate charge ordering in $\mathrm{La}_{0.5} \mathrm{Ca}_{0.5} \mathrm{MnO}_{3}$ using electron diffraction techniques. Our results suggest that this phenomenon may originate on the influence of $\mathrm{NN}-$ Coulomb interactions on double-exchange induced ferromagnetic phases of the manganites.

\section{ACKNOWLEDGMENTS}

A.L.M. acknowledges the financial support of the Conselho Nacional de Desenvolvimento Científico e Tecnológico (CNPq-Brazil), as well as partial support from the NHMFL In-House Research Program, supported under Grant No. DMR-9527035. S.Y. and E.D. were supported by the NSF Grant No. DMR-9520776.
*Present address: Departamento de Fìsica-UNESP/Bauru, Av. Eng. Luiz Edmundo Carrijo Coube, s/n. C. P. 473, Bauru SP 17033-360, Brazil.

${ }^{1}$ S. Jin et al., Science 264, 413 (1994), and references therein; see also J. M. D. Coey, M. Viret, and S. von Molnar, Adv. Phys. (to be published).

${ }^{2}$ C. Zener, Phys. Rev. 82, 403 (1951).

${ }^{3}$ A. P. Ramirez et al., Phys. Rev. Lett. 76, 3188 (1996); C. H. Chen and S-W. Cheong, ibid. 76, 4042 (1996); P. G. Radaelli, D. E. Cox, M. Marezio, and S.-W. Cheong, Phys. Rev. B 55, 3015 (1997); see also S-W. Cheong and C. H. Chen, in Colossal Magnetoresistance and Related Properties, edited by B. Raveau and C. N. R. Rao (World Scientific, Singapore, 1998).

${ }^{4}$ M. Hennion et al., Phys. Rev. Lett. 81, 1957 (1998).

${ }^{5}$ S. Yunoki, J. Hu, A. Malvezzi, A. Moreo, N. Furukawa, and E. Dagotto, Phys. Rev. Lett. 80, 845 (1998).

${ }^{6}$ E. Dagotto, S. Yunoki, A. L. Malvezzi, A. Moreo, J. Hu, S. Capponi, D. Poilblanc, and N. Furukawa, Phys. Rev. B 58, 6414 (1998).

${ }^{7}$ S. Yunoki and A. Moreo, Phys. Rev. B 58, 6403 (1998).

${ }^{8}$ S. Yunoki, A. Moreo, and E. Dagotto, Phys. Rev. Lett. 81, 5612 (1998); see also A. Moreo, S. Yunoki, and E. Dagotto, cond-mat/9901057 (unpublished).

${ }^{9}$ E. Dagotto, Rev. Mod. Phys. 66, 763 (1994), and references therein.

${ }^{10}$ A. J. Millis, P. B. Littlewood, and B. I. Shraiman, Phys. Rev. Lett. 74, 5144 (1995); H. Röder, J. Zang, and A. R. Bishop, ibid. 76, 1356 (1996); A. J. Millis, B. Shraiman, and R. Mueller, ibid. 77, 175 (1996).

${ }^{11}$ N. Furukawa, J. Phys. Soc. Jpn. 63, 3214 (1994).

${ }^{12}$ S. R. White, Phys. Rev. Lett. 69, 2863 (1992).

${ }^{13}$ J. Inoue and S. Maekawa, Phys. Rev. Lett. 74, 3407 (1995).

${ }^{14}$ P. G. de Gennes, Phys. Rev. 118, 141 (1960).

${ }^{15}$ E. L. Nagaev, Phys. Status Solidi B 186, 9 (1994); S-Q. Shen and Z. D. Wang, Phys. Rev. B 58, R8877 (1998); D. Arovas and F. Guinea, ibid. 58, 9150 (1998); H. Yi and J. Yu, ibid. 58, 11123 (1998); M. Yu. Kagan et al., cond-mat/9804213 (unpublished); M. Yamanaka, W. Koshibae, and S. Maekawa, Phys. Rev. Lett. 81, 5604 (1998).

${ }^{16}$ J. Riera, K. Hallberg, and E. Dagotto, Phys. Rev. Lett. 79, 713 (1997).

${ }^{17}$ G. Allodi et al., Phys. Rev. B 56, 6036 (1997); 57, 1024 (1998).

${ }^{18}$ J. W. Lynn et al., Phys. Rev. Lett. 76, 4046 (1996).

${ }^{19}$ J. M. De Teresa et al., Nature (London) 386, 256 (1997); see also 
J. B. Goodenough and J.-S. Zhou, ibid. 386, 229 (1997).

${ }^{20}$ D. E. Cox et al., Phys. Rev. B 57, 3305 (1998).

${ }^{21}$ Y. Yamada et al., Phys. Rev. Lett. 77, 904 (1996).

${ }^{22}$ Wei Bao et al., Solid State Commun. 98, 55 (1996).

${ }^{23}$ J. J. Rhyne, H. Kaiser, H. Luo, Gang Xiao, and M. L. Gardel, J. Appl. Phys. 93, 7339 (1998); M. Roy, J. F. Mitchell, A. P. Ramirez, and P. Schiffer, Phys. Rev. B 58, 5185 (1998); C. H. Booth, F. Bridges, G. H. Kwei, J. M. Lawrence, A. L. Cornelius, and J. J. Neumeier, Phys. Rev. Lett. 80, 853 (1998); Phys. Rev. B 57, 10440 (1998); M. Jaime, P. Lin, M. B. Salamon, P. Dorsey, and M. Rubinstein (unpublished); J.-S. Zhou and J. B. Goodenough, Phys. Rev. Lett. 80, 2665 (1998); S. J. L. Billinge, R. G. DiFrancesco, G. H. Kwei, J. D. Thompson, M. F. Hundley, and J. Sarrao (unpublished); M. R. Ibarra, G.-M. Zhao, J. M. De Teresa, B. Garcia-Landa, Z. Arnold, C. Marquina, P. A. Algarabel, H. Keller, and C. Ritter, Phys. Rev. B 57, 7446 (1998); R. H. Heffner et al., Phys. Rev. Lett. 77, 1869 (1996); H. L. Liu, S. L. Cooper, and S.-W. Cheong, ibid. 81, 4684 (1998).

${ }^{24}$ T. G. Perring et al., Phys. Rev. Lett. 78, 3197 (1997).

${ }^{25}$ In addition, J. Q. Li et al., Phys. Rev. Lett. 79, 297 (1997), reported phase segregation in $\mathrm{La}_{1-x} \mathrm{Sr}_{x} \mathrm{FeO}_{3}$.

${ }^{26}$ V. J. Emery and S. A. Kivelson, Physica C 209, 597 (1993); U. Löw, V. J. Emery, K. Fabricius, and S. A. Kivelson, Phys. Rev. Lett. 72, 1918 (1994); S. Haas, E. Dagotto, A. Nazarenko, and J. Riera, Phys. Rev. B 51, 5989 (1995).
${ }^{27}$ See also K. Kubo, J. Phys. Soc. Jpn. 51, 782 (1982); E. MüllerHartmann and E. Dagotto, Phys. Rev. B 54, R6819 (1996); J. Zang, H. Röder, A. R. Bishop, and S. A. Trugman, J. Phys.: Condens. Matter 9, L157 (1997); T. A. Kaplan and S. D. Mahanti, ibid. 9, L291 (1997); H. Röder, R. R. P. Singh, and J. Zang, Phys. Rev. B 56, 5084 (1997).

${ }^{28}$ B. Batlogg, S.-W. Cheong, and L. W. Rupp, Jr., Physica B 194196, 173 (1994); A. P. Ramirez et al., Phys. Rev. Lett. 72, 3108 (1994); J. F. DiTusa et al., ibid. 73, 1857 (1994).

${ }^{29}$ E. Dagotto, J. Riera, A. Sandvik, and A. Moreo, Phys. Rev. Lett. 76, 1731 (1996).

30 The ground state was always assumed to be even under reflections with respect to the center of the chain.

31 Note that strictly speaking at large $J_{H}$ and $n=1$ the system may resemble a spin-1 chain with a finite spin gap. However, even in this state the antiferromagnetic correlations are very robust. For the purposes of the present paper only the dominant character of the spin and charge correlations is important, and not their asymptotic behavior with distance.

${ }^{32}$ L. Sheng and C. S. Ting, Phys. Rev. B 57, 5265 (1998), and references therein.

${ }^{33}$ T. Mutou, N. Shibata, and K. Ueda, J. Phys.: Condens. Matter 10, L507 (1998).

${ }^{34}$ A. Luther and I. Peschel, Phys. Rev. B 12, 3908 (1975); D. M. Haldane, Phys. Rev. Lett. 45, 1358 (1980).

${ }^{35}$ C. H. Chen and S-W. Cheong, Phys. Rev. Lett. 76, 4042 (1996). 